\title{
MERGING BEAM-BEAM INTERACTION
}

\author{
Yuri K. Batygin and Takeshi Katayama \\ The Institute of Physical and Chemical Research (RIKEN), Saitama, 351-01, Japan
}

\section{Abstract}

New type of particle collisions is considered. In merging collisions two comoving beams intersect each other at small angle to provide low collision energy required for nuclear fusion experiments. Expression for luminosity is obtained. It is shown, that luminosity has a maximum value depending on particle velocity.

\section{INTRODUCTION}

Merging beam collisions is a new technique for study nuclear fusion processes [1]. Merging two radioactive isotope beams deliver low energy collisions just above the Coulomb barrier threshold that is difficult to be realized in other experimental methods. In this paper we analyze luminosity of merging beam-beam collision as a function of ring parameters and find optimal conditions to obtain maximum value of luminosity.

\section{LUMINOSITY OF MERGING BEAM- BEAM COLLISIONS}

Consider two bunched beams with particle densities $\rho_{1}, \rho_{2}$ and beam velocities $\vec{\beta}_{1}=\vec{v}_{1} / c, \vec{\beta}_{2}=\vec{v}_{2} / \mathrm{c}$, crossing each other with angle $2 \varphi$ (see Fig. 1). Assume beams have a Gaussian space charge density distributions

$$
\begin{gathered}
\rho_{1}=N_{1} \frac{\exp \left[-\frac{x_{1}^{2}}{2 \sigma_{\mathrm{x} 1}^{2}}-\frac{y_{1}^{2}}{2 \sigma_{\mathrm{y} 1}^{2}}-\frac{\left(\mathrm{z}_{1}-\mathrm{v}_{1} \mathrm{t}\right)^{2}}{2 \sigma_{\mathrm{z} 1}^{2}}\right]}{(2 \pi)^{3 / 2} \sigma_{\mathrm{x} 1} \sigma_{\mathrm{y} 1} \sigma_{\mathrm{z} 1}}, \\
\rho_{2}=\mathrm{N}_{2} \frac{\exp \left[-\frac{\mathrm{x}_{2}^{2}}{2 \sigma_{\mathrm{x} 2}^{2}}-\frac{\mathrm{y}_{2}^{2}}{2 \sigma_{\mathrm{y} 2}^{2}}-\frac{\left(\mathrm{z}_{2}-\mathrm{v}_{2} \mathrm{t}\right)^{2}}{2 \sigma_{\mathrm{z} 2}^{2}}\right]}{(2 \pi)^{3 / 2} \sigma_{\mathrm{x} 2} \sigma_{\mathrm{y} 2} \sigma_{\mathrm{z} 2}},
\end{gathered}
$$

where $\mathrm{N}_{1}, \mathrm{~N}_{2}$ are number of particles per each bunch, $\sigma_{\mathrm{xi}}, \sigma_{\mathrm{yi}}, \sigma_{\mathrm{zi}}, \mathrm{i}=1,2$ are root-mean square (rms) bunch sizes. Luminosity is defined as a ratio of interaction rate $\mathrm{dN} / \mathrm{dt}$ to cross section $\sigma$ of particle interaction $\mathrm{L}=(1 / \sigma)(\mathrm{dN} / \mathrm{dt})$. According to invariant cross section formula [2], number of collisions $\delta \mathrm{N}$ in the volume $\mathrm{dV}$ during time $\mathrm{dt}$ is

$$
\delta \mathrm{N}=\sigma \sqrt{\left(\overrightarrow{\mathrm{v}}_{1}-\overrightarrow{\mathrm{v}}_{2}\right)^{2}-\frac{\left[\overrightarrow{\mathrm{v}}_{1} \times \overrightarrow{\mathrm{v}}_{2}\right]^{2}}{\mathrm{c}^{2}}} \rho_{1} \rho_{2} \mathrm{dV} \mathrm{dt} .
$$

After two bunches crossed each other, total number of collisions, $\Delta \mathrm{N}$, is obtained via integration of Eq. (3) over volume of interacted bunches and time of interaction. In a ring $\mathrm{N}_{\mathrm{b}}$ bunches collide per turn during time $\mathrm{T}=1 / \mathrm{f}_{\mathrm{o}}$, where $f_{o}$ is a particle revolution frequency. Therefore, luminosity of particle collider, $\mathrm{L}=(1 / \sigma)(\Delta \mathrm{N} / \mathrm{T})$, is

$$
L=N_{b} f_{0} \sqrt{\left(\vec{v}_{1}-\vec{v}_{2}\right)^{2}-\frac{\left[\vec{v}_{1} \times \vec{v}_{2}\right]^{2}}{c^{2}}} \int_{V} \int_{t} \rho_{1} \rho_{2} d x d y d z d t .
$$

Transformation of coordinate system of every bunch $\left(\mathrm{x}_{\mathrm{i}}, \mathrm{y}_{\mathrm{i}}, \mathrm{z}_{\mathrm{i}}\right), \mathrm{i}=1,2$ into laboratory coordinate system $(\mathrm{x}, \mathrm{y}, \mathrm{z})$ is given by (see Fig. 1):

$$
\left\{\begin{array}{c}
\mathrm{z}_{1}=\mathrm{z} \cos \varphi+\mathrm{x} \sin \varphi \\
\mathrm{x}_{1}=-\mathrm{z} \sin \varphi+\mathrm{x} \cos \varphi
\end{array},\left\{\begin{array}{c}
\mathrm{z}_{2}=\mathrm{z} \cos \varphi-\mathrm{x} \sin \varphi \\
\mathrm{x}_{2}=\mathrm{z} \sin \varphi+\mathrm{x} \cos \varphi
\end{array}\right.\right.
$$

Substitution of coordinate transformation (5) into Eq. (4) gives the following expression for luminosity:

$$
L=G \frac{N_{b} f_{0} c N_{1} N_{2}}{(2 \pi)^{3}} \sqrt{\beta_{1}^{2}+\beta_{2}^{2}-2 \beta_{1} \beta_{2} \cos 2 \varphi-\beta_{1}^{2} \beta_{2}^{2} \sin ^{2} 2 \varphi},
$$

where integral over volume and time of interaction is

$$
\begin{aligned}
& G=\int_{-\infty}^{\infty} \int_{-\infty}^{\infty} \int_{-\infty}^{\infty} \int_{-\infty}^{\infty} \frac{1}{\sigma_{\mathrm{x} 1} \sigma_{\mathrm{y} 1} \sigma_{\mathrm{z} 1} \sigma_{\mathrm{x} 2} \sigma_{\mathrm{y} 2} \sigma_{\mathrm{z} 2}} . \\
& \exp \left\{-\mathrm{x}^{2}\left[\frac{\cos ^{2} \varphi}{2}\left(\frac{1}{\sigma_{\mathrm{x} 1}^{2}}+\frac{1}{\sigma_{\mathrm{x} 2}^{2}}\right)+\frac{\sin ^{2} \varphi}{2}\left(\frac{1}{\sigma_{\mathrm{z} 1}^{2}}+\frac{1}{\sigma_{\mathrm{z} 2}^{2}}\right)\right]\right. \\
& -\frac{\mathrm{y}^{2}}{2}\left(\frac{1}{\sigma_{\mathrm{y} 1}^{2}}+\frac{1}{\sigma_{\mathrm{y} 2}^{2}}\right)-\mathrm{z}^{2}\left[\frac{\sin ^{2} \varphi}{2}\left(\frac{1}{\sigma_{\mathrm{x} 1}^{2}}+\frac{1}{\sigma_{\mathrm{x} 2}^{2}}\right)\right.
\end{aligned}
$$

$\left.+\frac{\cos ^{2} \varphi}{2}\left(\frac{1}{\sigma_{\mathrm{z} 1}^{2}}+\frac{1}{\sigma_{\mathrm{z} 2}^{2}}\right)\right]-\mathrm{z} \mathrm{t} \cos \varphi\left(-\frac{\mathrm{v}_{1}}{\sigma_{\mathrm{z} 1}^{2}}-\frac{\mathrm{v}_{2}}{\sigma_{\mathrm{z} 2}^{2}}\right)$

$$
-\frac{\mathrm{t}^{2}}{2}\left(\frac{\mathrm{v}_{1}^{2}}{\sigma_{\mathrm{z} 1}^{2}}+\frac{\mathrm{v}_{2}^{2}}{\sigma_{\mathrm{z} 2}^{2}}\right)+\mathrm{x}\left[\mathrm{z} \sin \varphi \cos \varphi\left(\frac{1}{\sigma_{\mathrm{x} 1}^{2}}-\frac{1}{\sigma_{\mathrm{x} 2}^{2}}-\frac{1}{\sigma_{\mathrm{z} 1}^{2}}+\frac{1}{\sigma_{\mathrm{z} 2}^{2}}\right)\right.
$$

$\left.\left.-\mathrm{t} \sin \varphi\left(\frac{\mathrm{V}_{1}}{\sigma_{\mathrm{z} 1}^{2}}-\frac{\mathrm{V}_{2}}{\sigma_{\mathrm{z} 2}^{2}}\right)\right]\right\} d \mathrm{dx} d \mathrm{dz} d \mathrm{t}$.

Assume, that collide bunches have the same longitudinal sizes $\sigma_{\mathrm{z} 1}=\sigma_{\mathrm{z} 2}=\sigma_{\mathrm{z}}$ and same velocities $\beta_{1}=\beta_{2}=\beta$, but different transverse sizes $\sigma_{\mathrm{x} 1} \neq \sigma_{\mathrm{x} 2}, \quad \sigma_{\mathrm{y} 1} \neq \sigma_{\mathrm{y} 2}$. Calculation of luminosity, Eqs.(6-7) results in expression 


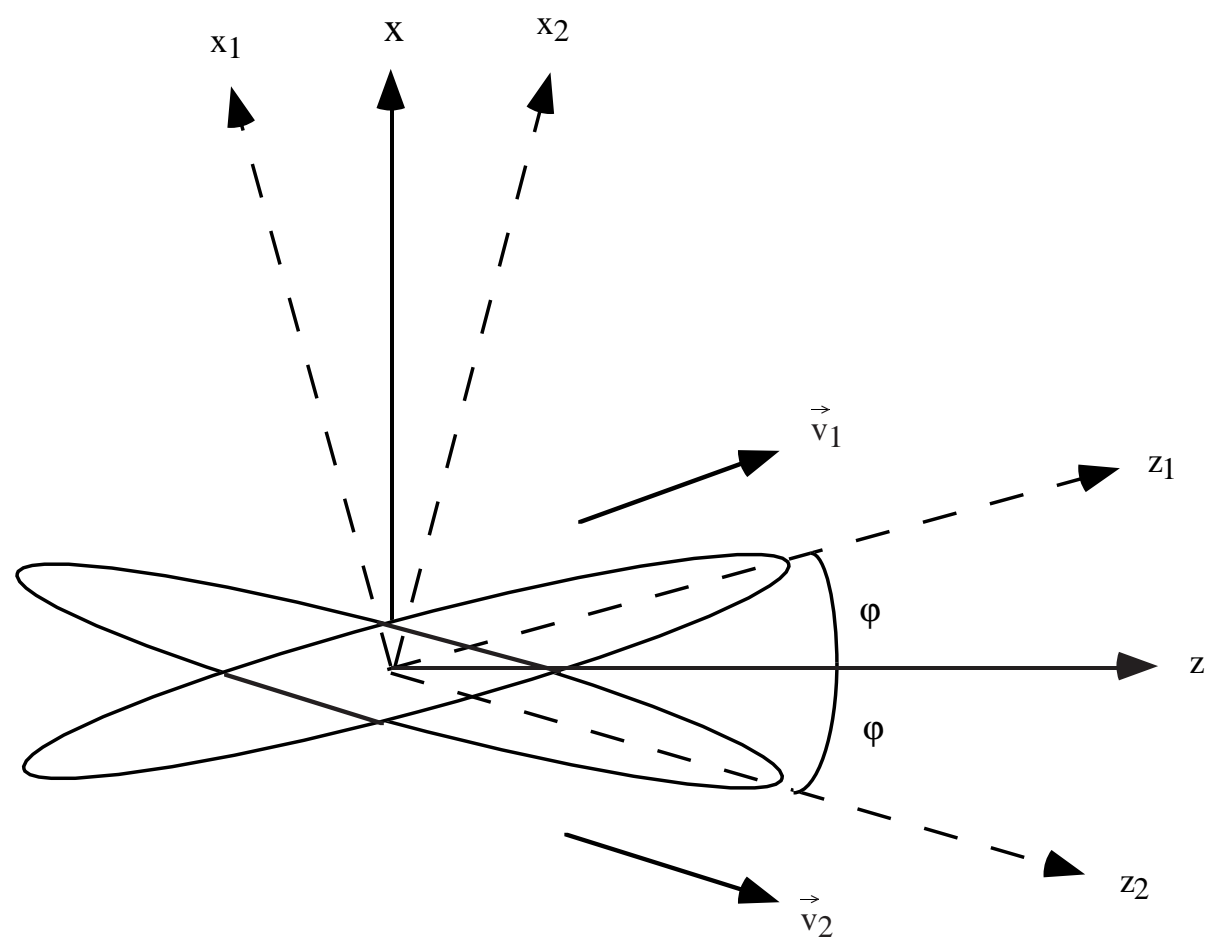

Fig. 1. Merging beam-beam interaction.

$\mathrm{L}=\frac{\mathrm{N}_{\mathrm{b}} \mathrm{f}_{\mathrm{o}} \mathrm{N}_{1} \mathrm{~N}_{2}}{2 \sqrt{2} \pi \sqrt{\sigma_{\mathrm{y} 1}^{2}+\sigma_{\mathrm{y} 2}^{2}} \sigma_{\mathrm{z}} \cos \varphi} \sqrt{\frac{1-\beta^{2} \cos ^{2} \varphi}{1+\operatorname{tg}^{2} \varphi\left(\frac{\sigma_{\mathrm{x} 1}^{2}+\sigma_{\mathrm{x} 2}^{2}}{\sigma_{\mathrm{z}}^{2}}\right)}}$.

Formula (8) is derived assuming $\varphi \neq 0, \pi / 2$. For typical parameters in the interaction point, $\varphi<<2 \pi$, $\sigma_{\mathrm{x} 1,2} \ll \sigma_{\mathrm{z}}$, the denominator factor in Eq. (8) can be neglected as a product of two small values:

$$
\operatorname{tg}^{2} \varphi\left(\frac{\sigma_{\mathrm{x} 1}^{2}+\sigma_{\mathrm{x} 2}^{2}}{\sigma_{\mathrm{z}}^{2}}\right)<<1 .
$$

Luminosity, Eq.(8), is inversely proportional to longitudinal beam size, $\sigma_{\mathrm{z}}$, and vertical beam size, $\sigma_{\mathrm{y}} 1,2$, and practically independent on horizontal beam sizes in the plane of particle collision, $\sigma_{\mathrm{x} 1,2}$.

Let us take into account, that revolution frequency of particle motion in a ring is given by $f_{o}=\beta c / C_{r}$ where $C_{r}=2 \pi R$ is a circumference and $R$ is a mean radius of the ring. Therefore, luminosity as a function of particle velocity is:

$$
L=\frac{c N_{b} N_{1} N_{2}}{2 \sqrt{2} \pi \cos \varphi \sqrt{\sigma_{\mathrm{y} 1}^{2}+\sigma_{\mathrm{y} 2}^{2}} \sigma_{\mathrm{z}} \mathrm{C}_{\mathrm{r}}} \beta \sqrt{1-\beta^{2} \cos ^{2} \varphi} .
$$

Taking derivative of luminosity over particle velocity,

$$
\frac{\partial \mathrm{L}}{\partial \beta}=\frac{\mathrm{c} \mathrm{N} \mathrm{N}_{1} \mathrm{~N}_{2}}{2 \sqrt{2} \pi \cos \varphi \sqrt{\sigma_{\mathrm{y} 1}^{2}+\sigma_{\mathrm{y} 2}^{2}} \sigma_{\mathrm{z}} \mathrm{C}_{\mathrm{r}}} \frac{\left(1-2 \beta^{2} \cos ^{2} \varphi\right)}{\sqrt{1-\beta^{2} \cos ^{2} \varphi}}=0,
$$

the maximum luminosity, $\mathrm{L}_{\max }$, is obtained at the following optimal values of particle velocity, $\beta_{\text {opt }}$, and particle energy, $\gamma_{\text {opt }}$ :

$$
\begin{gathered}
\beta_{\mathrm{opt}}=\frac{1}{\sqrt{2} \cos \varphi}, \quad \gamma_{\mathrm{opt}}=\frac{\sqrt{2} \cos \varphi}{\sqrt{2 \cos ^{2} \varphi-1}}, \\
\mathrm{~L}_{\max }=\frac{\mathrm{c} \mathrm{N}_{\mathrm{b}} \mathrm{N}_{1} \mathrm{~N}_{2}}{4 \sqrt{2} \pi \cos ^{2} \varphi \sqrt{\sigma_{\mathrm{y} 1}^{2}+\sigma_{\mathrm{y} 2}^{2}} \sigma_{\mathrm{z}} \mathrm{C}_{\mathrm{r}}}
\end{gathered}
$$

In Fig. 2 luminosity as a function of particle velocity for angle $\varphi=0.087$ is presented. Since the value of $\varphi$ is small and $\cos \varphi \approx 1$, the maximum luminosity is obtained for $\beta_{\mathrm{opt}} \approx 1 / \sqrt{2}, \gamma_{\mathrm{opt}} \approx 1.41$. At relativistic energies, $\beta \approx 1$, luminosity is

$$
\mathrm{L}(\beta \rightarrow 1)=\frac{\mathrm{c} \mathrm{N}_{\mathrm{b}} \mathrm{N}_{1} \mathrm{~N}_{2}}{2 \sqrt{2} \pi \sqrt{\sigma_{\mathrm{y} 1}^{2}+\sigma_{\mathrm{y} 2}^{2}} \sigma_{\mathrm{z}} \mathrm{C}_{\mathrm{r}}} \operatorname{tg} \varphi .
$$

Therefore, reduction of luminosity at relativistic particle energies $\gamma \gg \gamma_{o p t}$ with respect to maximum value of luminosity is

$$
\frac{\mathrm{L}(\beta \rightarrow 1)}{\mathrm{L}_{\max }\left(\beta_{\mathrm{opt}}\right)}=\sin 2 \varphi
$$




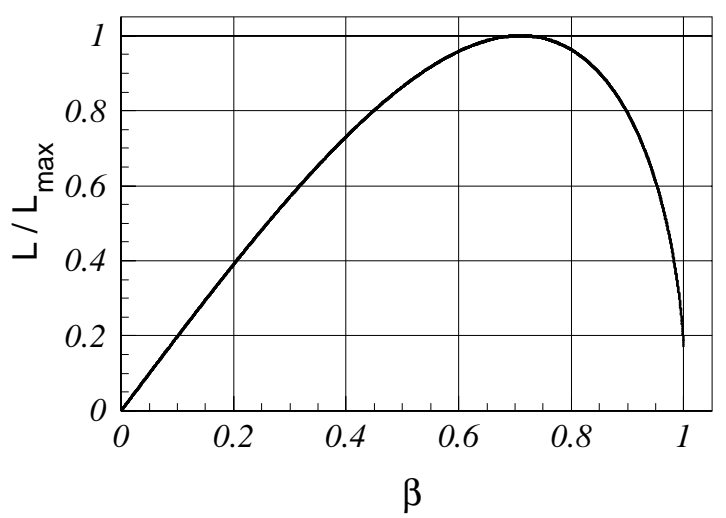

Fig. 2. Luminosity of merging beam-beam collisions for angle $\varphi=0.087$ as a function of particle velocity.

\section{COMPARISON WITH LUMINOSITY OF CROSSING ANGLE COLLISIONS}

Let us compare maximum luminosity at merging collisions, Eq. (13) with that for head crossing angle collision with the same value of $\varphi$. Expression for luminosity with head crossing angle collision is obtained from general expression, Eqs. (6), (7), by change of $\vec{v}_{2}$ for $-\vec{v}_{2}[3]$ :

$$
L=\frac{N_{b} f_{o} N_{1} N_{2} \sqrt{1-\beta^{2} \sin ^{2} \varphi}}{2 \pi \sqrt{\sigma_{\mathrm{y} 1}^{2}+\sigma_{\mathrm{y} 2}^{2}} \sqrt{2 \sigma_{\mathrm{z}}^{2} \sin ^{2} \varphi+\cos ^{2} \varphi\left(\sigma_{\mathrm{x} 1}^{2}+\sigma_{\mathrm{x} 2}^{2}\right)}} .
$$

Ratio of two values of luminosity is:

$$
\begin{aligned}
\frac{L_{\text {merge max }}}{L_{\text {cross angle }}} & =\frac{\sqrt{\sigma_{\mathrm{x} 1}^{2}+\sigma_{\mathrm{x} 2}^{2}}}{2 \sqrt{2} \sigma_{\mathrm{z}} \beta \cos \varphi} \sqrt{\frac{1+\operatorname{tg}^{2} \varphi \frac{2 \sigma_{\mathrm{z}}^{2}}{\left(\sigma_{\mathrm{x} 1}^{2}+\sigma_{\mathrm{x} 2}^{2}\right)}}{1-\beta^{2} \sin ^{2} \varphi}} \\
& \approx \frac{\sigma_{\mathrm{x}}}{2 \sigma_{\mathrm{z}}} .
\end{aligned}
$$

For typical ring parameters $\varphi \approx 0, \quad \beta \approx 1$, ratio of luminosity is of the order $\sigma_{\mathrm{x}} / \sigma_{\mathrm{z}} \approx 10^{-3}$. That means, that in merging collisions, achievable value of luminosity is several order of magnitude less than that in encountered crossing angle collisions, because merge beams are almost parallel to each other.

\section{LUMINOSITY OF COMOVING BEAM COLLISIONS}

From the previous section it is clear that merging collision is characterized by small value of luminosity. V.V.Parhomchuk (BINP, Novosibirsk) pointed out [4] that, if collisions is performed on-axis, luminosity does not depend on particle velocities. Consider two bunched beams, which move in the same direction with different velocities $\mathrm{v}_{1} \neq \mathrm{v}_{2}$. Luminosity of such comoving collisions is obtained from general formulas (6), (7) by change of $\vec{v}_{2}$ for $-\vec{v}_{2}$ and assuming $\varphi=0$ :

$$
\begin{aligned}
& L=\frac{N_{b} f_{o} c N_{1} N_{2}}{(2 \pi)^{3}} \frac{\left|\beta_{1}-\beta_{2}\right|}{\sigma_{x}^{2} \sigma_{y}^{2} \sigma_{z}^{2}} \cdot G \\
& G=\int_{-\infty}^{\infty} \int_{-\infty}^{\infty} \int_{-\infty}^{\infty} \int_{-\infty}^{\infty} \exp \left[-\frac{x^{2}}{\sigma_{x}^{2}}-\frac{y^{2}}{\sigma_{y}^{2}}-\frac{z^{2}}{\sigma_{z}^{2}}-\frac{t^{2}}{2}\left(\frac{v_{1}^{2}+v_{2}^{2}}{\sigma_{z}^{2}}\right)\right. \\
&\left.+z \cdot t \frac{\left(v_{1}+v_{2}\right)}{\sigma_{z}^{2}}\right] d x d y d z d t
\end{aligned}
$$

After evaluation of integral (19), the value of luminosity is:

$$
L=\frac{N_{b} f_{o} N_{1} N_{2}}{4 \pi \sigma_{x} \sigma_{y}}
$$

Eq. (20) gives the same result as luminosity of headon collision. Integration in Eq. (19) is performed in the infinite limits. It means, that bunches of both beams have to pass through each other completely. Bunch length is $l \approx 4 \sigma_{\mathrm{z}}$. To perform interaction, collision region, $l_{\text {coll }}$, has to be as long as [4]

$$
l_{\text {coll }}=l \frac{\beta_{2}}{\left|\beta_{1}-\beta_{2}\right|} .
$$

Typical value of ratio in Eq. (21) is $\beta_{2} / \Delta \beta \approx 10$. To obtain the same value of luminosity in comoving collisions as that for head-on collisions, it is necessary to provide long interaction region with short bunches [4]. Merging beam collisions deliver smaller value of luminosity with short interaction region.

\section{REFERENCES}

[1] T.Katayama, Y.Batygin, N.Inabe, K.Ohtomo, T.Ohkawa, M.Takanaka, M.Wakasugi, S.Watanabe, Y.Yano, K.Yoshida, J.Xia, Y.Rao and Y.Yuan, Nuclear Physics A626 (1997), 545560.

[2] L.Landau and E.Lifshitz, The Classical Theory of Fields, Pergamon Press, 1975.

[3] Y.Batygin and T.Katayama, Luminosity of particle collider, RIKEN-AF-AC-10, 1998.

[4] V.V.Parhomchuk, Private communication, 1998. 